\title{
The efficacy of collagen based dressings for treatment of scald burns in under 5 years paediatric population: a non-comparative, prospective single centre experience
}

\author{
Mohammed Fahud Khurram, Mohd Tafazul Sheikh*, Imran Ahmad, \\ Sudheer Kumar Maurya, Vivek Ambedkar, Rajesh Kumar Maurya
}

Department of Plastic and Reconstructive Surgery, JNMCH, AMU, Aligarh, Uttar Pradesh, India

Received: 05 June 2018

Revised: 13 November 2018

Accepted: 14 November 2018

*Correspondence:

Dr. Mohd Tafazul Sheikh,

E-mail: drtafazuls@gmail.com

Copyright: () the author(s), publisher and licensee Medip Academy. This is an open-access article distributed under the terms of the Creative Commons Attribution Non-Commercial License, which permits unrestricted non-commercial use, distribution, and reproduction in any medium, provided the original work is properly cited.

\section{ABSTRACT}

Background: One of the major challenges faced worldwide is management of burn wounds, particularly among the pediatric population. Immense research is going on to overcome the challenges associated with survival of these patients and healing of burnt areas. Newer dressing materials are being developed and one of them is collagen. The collagen is biocompatible. The aim was to achieve earlier and better healing of wounds, and to obviate the need for frequent change of dressings; which besides being painful, is quite troublesome in pediatric population.

Methods: A prospective study was conducted in 38 patients of scald burns under-5 years of age, from October 2016 till July 2017. After thorough cleaning of burn wounds and thorough rinsing of collagen sheets with normal saline, the wounds were covered with sheets of collagen. Paraffin with chlorhexidine impregnated gauze dressing was applied over the sheet of collagen followed by dry gauze, cotton and bandage application. First dressing was opened after 5 days and then after 3 to 4 days.

Results: The wounds healed in 10 to 14 days in most of them. Infection developed in one case, warranting removal of collagen sheet. None developed any adverse reaction to the application of collagen dressing.

Conclusions: We conclude that collagen based dressing is a very good modality of treatment for the burn wounds with advantages of being cost effective, requiring lesser number of dressings and blood transfusions, and shorter duration of hospital stay.

Keywords: Pediatric burns, Scald burn, Collagen

\section{INTRODUCTION}

Burn injuries continue to be one of the major challenges faced worldwide, more so in the developing countries and particularly among the pediatric population. Due to the susceptibility of burn wounds, colonization by opportunistic organisms can take place. Besides microbial factors, the likelihood of invasive burn wound infection is also determined by the age of patient and the extent and depth of burn. In developing countries, the sepsis related mortality associated with burn injuries is about $75 \% .^{1}$

Usually small surface areas are involved due to burns in children and majority belongs to the under 5 year age group. In them scald burns, especially due to spillage of hot drinks, is found to be the major etiological factor. ${ }^{2}$

Healing in a burn wound is a natural process in a given favorable environment. Changes of dressings which are 
not non-adherent, traumatize the newly forming tissues leading to personal suffering and delay in healing in addition to the psychological suffering. ${ }^{3}$

Covering of superficial thermal burn wounds by collagen sheets is a common practice in the present times. By applying it over the wound, exposed dermis and free nerve endings get covered leading to reduction in pain and infection. ${ }^{4}$ Also, it acts as an interface between the wound's internal milieu and the external environment. Collagen sheet application is an easy, simple and painless procedure besides being natural, hypoallergenic, and nonpyrogenic. ${ }^{5}$

\section{Aims of study}

The aim was to achieve earlier and better healing of wounds, and to obviate the need for frequent change of dressings; which besides being painful, is quite troublesome in pediatric population which comprises of around $12 \%$ (in under-5 year age group) of the total burn patients who present in our hospital.

\section{METHODS}

A prospective study was conducted in 38 patients of scald burns under-5 years of age, from 1st October 2016 till the end of July 2017, at Jawaharlal Nehru Medical College Hospital, Aligarh Muslim University, Aligarh, which is a tertiary care hospital, after obtaining ethical approval from the Department of Plastic Surgery and the institution. Patients presenting after first 24 hours of burn injury and those having more than $30 \%$ TBSA burns were excluded from the study. In all the cases, wet sheets of Collagen were applied over the wounds. On arrival of the patient in the Emergency Department or in the Out Patient Department (OPD), thorough assessment of the patient was routinely carried out as per the ATLS protocol. Total body surface area (TBSA) percentage was assessed by using Lund and Browder Chart. Also the depth and areas affected were noted. Then before starting the dressing, thorough cleansing of the affected areas (with luke warm Normal Saline) was carried out which included removal of the foreign material or removal of different types of compounds that had been applied over the burn areas before presenting in the hospital. Under aseptic conditions and after ensuring prevention of hypothermia, the blisters were excised till the wound was free of any non-viable tissue (where required). This was followed by thorough rinsing of collagen sheets with normal saline so as to remove the preservative (isopropyl alcohol). The wounds were covered with sheets of collagen depending upon the percentage of total body surface area (TBSA) involved. The sizes of sheets used in the patients were $10 \times 10 \mathrm{~cm}^{2}$ and $10 \times 15 \mathrm{~cm}^{2}$, which were trimmed (if needed) as per the requirement. The placement of sheets over the wounds was done in such a way so as to ensure the coverage of entire wound area and removal of all trapped air bubbles beneath the sheet. Then paraffin gauze dressing, impregnated with chlorhexidine, was applied over the sheet of collagen followed by sequential application of dry gauze, cotton and bandage. The patients with more than $10 \%$ TBSA burns or having burns over face, hands; were admitted in the hospital and rest were discharged from the hospital with an advice to follow up in the OPD. Collagen sheet dressing was not applied over the wounds present on face and neck, if any. Antibiotic prophylaxis and analgesic syrup was given to all the patients and Tetanus prophylaxis provided in the non-immunized ones. First dressing was opened after 5 days of application over the wounds.

While opening the first dressing, only superficial layers, i.e. up to the dry gauze layer, were removed and wounds were inspected for any kind of problem like soakage, collection, foul smell, etc. After inspecting the wound and finding it satisfactory, a layer of ointment povidine iodine was applied and the wound redressed with a layer of dry gauze, followed by cotton layer and finally bandage application. Similar process was repeated 3-4 days later and the redundant peeled off areas of collagen and paraffin gauze were trimmed. Then, if needed, wounds were dressed up again in similar fashion but with lighter layers of cotton and bandage or otherwise left open.

\section{RESULTS}

There were 16 male patients and 22 female patients which included three patients less than 1 year old, six patients in the age group of 1-2 years, nine patients in the age group of 2- 3 years, eight patients in the age group of 3-4 years and twelve patients in the age group of $4-<5$ years.

Table 1: Gender distribution.

\begin{tabular}{|lll|}
\hline Gender & Number & Percentage (\%) \\
\hline Male & 16 & 42.10 \\
\hline Female & 22 & 57.89 \\
\hline
\end{tabular}

Table 2: Age-wise distribution of patients.

\begin{tabular}{|lll|}
\hline $\begin{array}{l}\text { Age group } \\
\text { (in years) }\end{array}$ & Number & Percentage $(\%)$ \\
\hline$<\mathbf{1}$ & 3 & 07.89 \\
\hline $\mathbf{1}-<\mathbf{2}$ & 6 & 15.78 \\
\hline $\mathbf{2}-<\mathbf{3}$ & 9 & 23.68 \\
\hline $\mathbf{3}-<\mathbf{4}$ & 8 & 21.05 \\
\hline $\mathbf{4 - < 5}$ & 12 & 31.57 \\
\hline
\end{tabular}

Out of thirty eight patients, thirty six patients had superficial second degree burn injuries and two patients had deep burn injuries; wherein eight patients had involvement of trunk only, fourteen patients had involvement of trunk and lower limbs, ten patients had involvement of trunk and upper limbs and six patients had involvement of face, neck and trunk. All were scald 
burns; eleven affected with hot milk, fifteen affected with hot water, six affected with hot tea, four affected with hot liquid food and two with hot oil. Twenty two patients were discharged after presentation on the same day. Sixteen patients were hospitalized. Twenty two patients presented with up to $10 \%$ scald burns, five patients had up to $15 \%$ scald burns, three patients had up to $20 \%$ scald burns, six patients had up to $25 \%$ scald burns and two patient had $30 \%$ scald burns.

Table 3: Distribution of patients as per body areas affected due to burns.

\begin{tabular}{|lll|}
\hline Body areas affected & Number & Percentage (\%) \\
\hline Trunk only & 8 & 21.05 \\
\hline Trunk and lower limbs & 14 & 36.84 \\
\hline Trunk and upper limbs & 10 & 26.31 \\
\hline Trunk, face and neck & 6 & 15.78 \\
\hline
\end{tabular}

Table 4: Patient distribution in terms of burn etiology.

\begin{tabular}{|lll|}
\hline Etiology & Number & Percentage (\%) \\
\hline Hot milk & 11 & 28.94 \\
\hline Hot water & 15 & 39.47 \\
\hline Hot tea & 6 & 15.78 \\
\hline Hot liquid food & 4 & 10.52 \\
\hline Hot oil & 2 & 05.26 \\
\hline
\end{tabular}

Out of sixteen patients who were admitted, eight patients were discharged on the 5th day after changing the first dressing and two patients were discharged on the 8th day after changing the second dressing, with an advice to follow up in the OPD. Three patients were discharged on the 12th day after removing the dressing. Three patients

required prolonged admission i.e. more than 12 days; one among them was discharged on the 15 th day (injury due to hot liquid food), one on 18th day (injury due to hot oil), and one patient, who had sustained around $25 \%$ scald burn (injury due to hot oil), had developed infection which warranted complete removal of collagen sheets on the 5th day and underwent daily changing of conventional dressing till discharge from the hospital on the 21st day. The patient whose collagen dressing needed removal, was transfused one unit of PRBC (in three aliquots) on three consecutive days, underwent debridement with STSG and was discharged on $21^{\text {st }}$ day after burn injury. All the patients who were discharged on day 1 of presentation and on day 5 and day 8 of admission had their wounds healed by 10th day. Among those who were admitted in the hospital, the wounds of three patients were healed by day $12 \mathrm{th}$. Out of the 3 patients who required prolonged admission (i.e. more than 12 days), one patient's wounds healed by 15 th day and the other patient's wounds healed by 18 th day. In one case, split thickness skin grafting was done. The wounds healed in 10 to 14 days in most of the study patients. None of the patients in our study developed any adverse reaction to the application of collagen dressing.

Table 5: Distribution of patients in terms of total body surface area (TBSA) burns.

\begin{tabular}{|lll|}
\hline TBSA burns & Number & Percentage (\%) \\
\hline $\mathbf{0 -}<\mathbf{1 0 \%}$ & 22 & 57.89 \\
\hline $\mathbf{1 0}-<\mathbf{1 5 \%}$ & 5 & 13.15 \\
\hline $\mathbf{1 5}-<\mathbf{2 0 \%}$ & 3 & 07.89 \\
\hline $\mathbf{2 0}-<\mathbf{2 5 \%}$ & 6 & 15.78 \\
\hline $\mathbf{2 5}-\mathbf{3 0 \%}$ & 2 & 05.26 \\
\hline
\end{tabular}
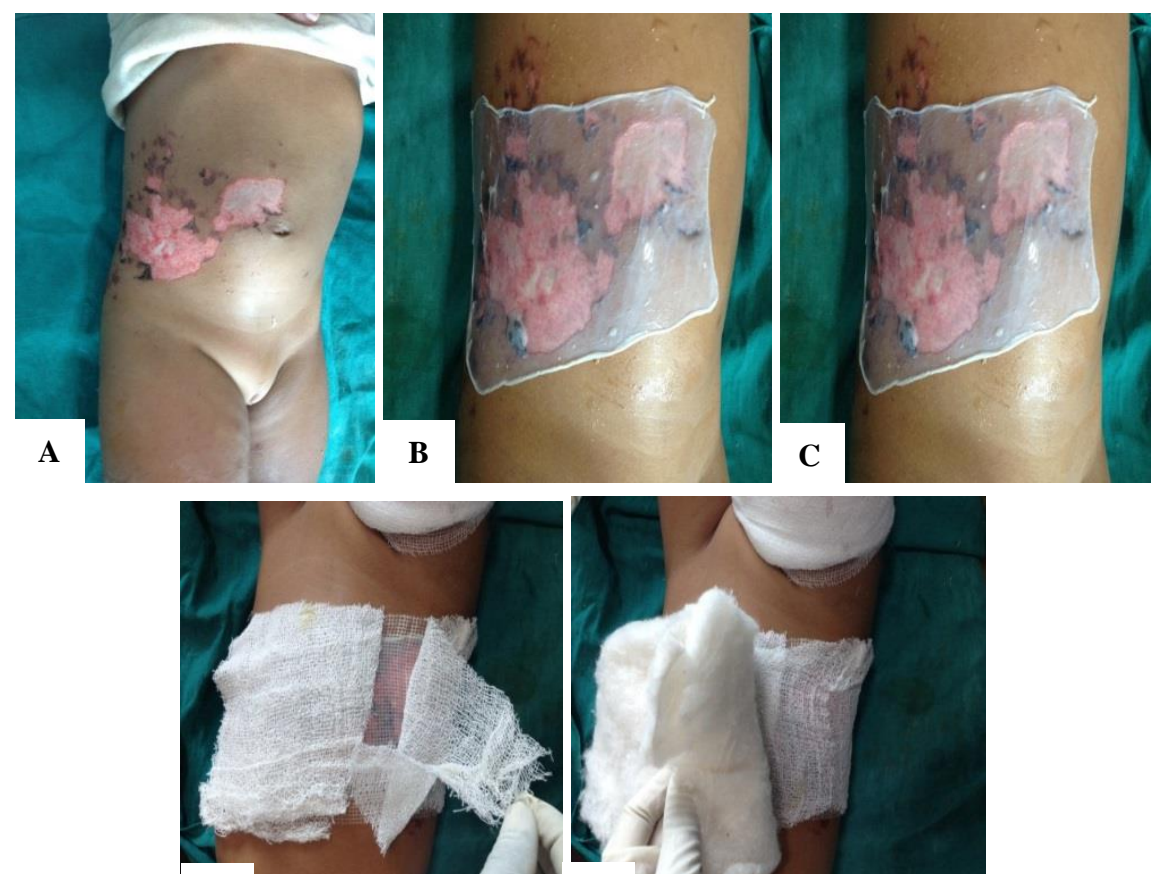

D
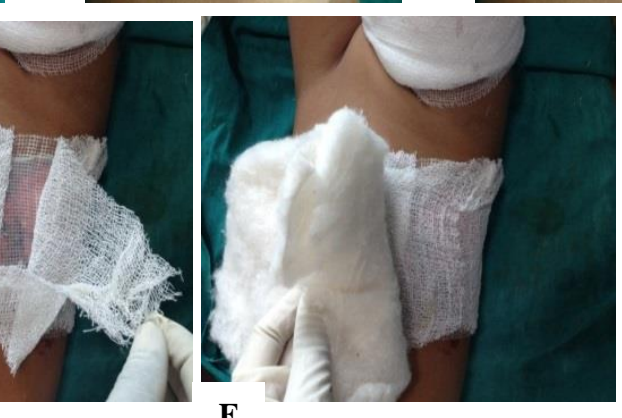

$\mathbf{E}$

Figure 1: (A): At the time of presentation; (B): after collagen sheet application; $(C)$ : after paraffin gauze application; (D): after plain gauze application; (E): after layer of cotton application. 

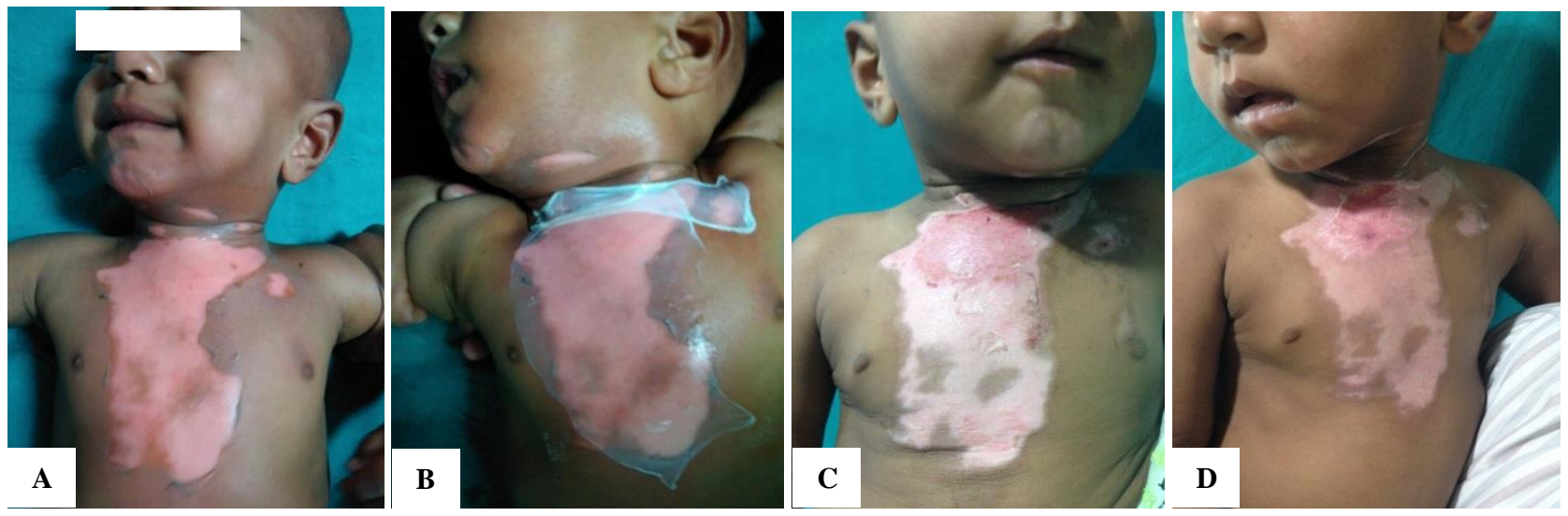

Figure 2: (A): At the time of presentation; (B): after collagen sheet application; (C): presentation on day 8; (D): presentation on day 12 .
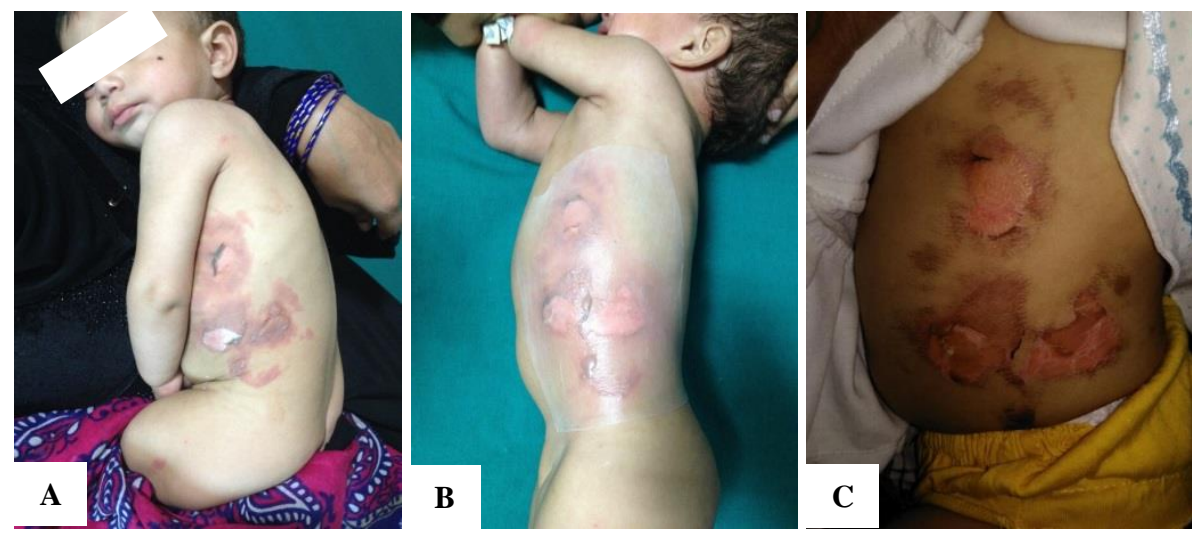

Figure 3: (A): At the time of presentation; (B): after collagen sheet application; (C): presentation on day 10.

\section{DISCUSSION}

Out of total burn admissions in India, 17-25\% account for pediatric burns with household accidents being the cause in about $90 \%$ of cases. ${ }^{6}$ Collagen sheets, as a form of dressing, have great utility in the management of various forms of wounds of varied etiology including burns. It has eased out the wound management and acted as an effective alternative modality for wound care.

Bovine collagen sheets are used as dressing, as it has similarity to human collagen, after it undergoes technical/procedural processing and sterilization. ${ }^{7}$ By the activity of inflammatory cells, it gets absorbed gradually after being retained in the tissue. Practically, due to its abundant occurrence in nature, collagen is being prepared easily. Also, essentially collagen degrades in biological environments without leaving any foreign residue. Key to its usage as a biomaterial is its solubility without destroying its basic rigid triple helical structure. ${ }^{8}$ Action of metalloproteinase is inhibited by collagen dressing usage. ${ }^{9}$ Studies suggest superior wound healing and pain control with collagen dressing. ${ }^{5,10}$ Moist wound environment is maintained by collagen dressings which is required for improved wound healing. It, besides promoting angiogenesis, increases the repair mechanisms pertaining to body and through deposition and organization of freshly formed fibers encourages wound healing in addition to prevention of dehydration and protection from mechanical injury. ${ }^{4}$ Healing may get accelerated with reduction of hypertrophic scarring on using biological dressing for the protection of wound. ${ }^{11}$

Shettigar et al in their study on animal models, showed that the collagen dressed burn wounds had faster epithelialization with dense fibrous tissue in growth in the subdermal layers and little inflammatory change. ${ }^{12}$ In a study done by Veves and Sheehan on 276 patients with diabetic foot wounds, wherein two equal groups were compared; one group treated with collagen and second with a different material, found better healing in wounds of less than six months duration. ${ }^{9}$ A significant increase in healing rate was demonstrated by Gao et al, in their study. ${ }^{13}$ Lazovic et al conducted a study on the application of collagen in open wound healing wherein they concluded that not only collagen is very fruitful in first and second degree burns, but also it is economical and avoids pain associated with dressing, particularly in children. ${ }^{14}$ Waghmare et al in their study on 100 children with partial thickness burns found that on using the collagen dressing there was no complication in $92 \%$ of patients with good healing at 7-10 days. There were 
minor complications in only $8 \%$ of patients. They concluded that in children with first and second degree burns, usage of collagen sheet for dressing is very beneficial as it is well tolerated and has fewer complications, besides avoiding pain and traumatic experience of conventional dressings. ${ }^{2}$

In our study we noted an outcome with the collagen sheet dressings which were very encouraging in terms of it being a relatively painless procedure and achievement of early wound healing besides minimizing the traumatic experience of frequent dressing changes as in case of conventional dressing. Our results fall in line with the results of various other studies. We did not notice any adverse effect of the collagen sheet.

\section{CONCLUSION}

We conclude that collagen based dressing is a very good modality of treatment for the burn wounds with advantages of being cost effective and can be done as a part of first aid requiring no special needs. Burnt area requires lesser number of dressings, patients need lesser blood transfusions and have shorter duration of hospital stay. Also, it minimizes the psychological trauma of both, the pediatric patients as well as their parents, by the need for change of lesser number of dressings; which is more painful for the parents on seeing their children in agony than the pain of the affected children itself.

Funding: No funding sources Conflict of interest: None declared

Ethical approval: The study was approved by the Institutional Ethics Committee

\section{REFERENCES}

1. Khan TS, Bijli AH, Wani AH. Microbiological and quantitative analysis of burn wounds in the burn unit at a tertiary care hospital in Kashmir. Indian $\mathbf{J}$ Burns. 2016;24:62-8.

2. Waghmare M, Shah H, Tiwari C, Makhija D, Desale $\mathrm{J}$, Dwivedi P. Collagen dressings in the management of partial thickness pediatric burns: Our experience. Indian J Burns. 2016;24:53-7.

3. Vartak AM. Care of the burn wound: As I do it. Indian J Burns. 2016;24:3-7.

4. Ramesh BA, Jayalakshmi BK, Mohan J. A comparative study of collagen dressing versus petrolatum gauze dressing in reducing pain at the donor area. J Cutan Aesthet Surg. 2017;10:18-21.

5. Singh O, Gupta SS, Soni M, Moses S, Shukla S, Mathur RK. Collagen dressing versus conventional dressings in burn and chronic wounds: A retrospective study. J Cutan Aesthet Surg. 2011;4:12-6.

6. Kumar R, Parashar A. Special consideration in pediatric burn patients; Indian $\mathrm{J}$ Plast Surg. 2010;43(1):43-50.

7. Marriappan N. Collagen dressing for thermal burns. Sch J App Med Sci. 2015;3(1):58-61.

8. Stenzel KH, Miyata T, Rubin AL. Collagen as a biomaterial. Ann Rev Biophys Bioeng. 1974;3:23153.

9. Veves A, Sheehan P, Pham HT. A randomized, controlled trial of promogran (a collagen/oxidized regenerated cellulose dressing) vs standard treatment in the management of diabetic foot ulcers. Arch Surg. 2002;137:822-7.

10. Sreekumar NC, Bhandari PL, Praveen N. Comparative study of collagen and paraffin gauze dressing on skin graft donor site. Indian J Burns. 2015;23:81-3.

11. Rai R, Sudarshan SH, Dsouza R, Saldhana E, Aithala PS. Collagen dressing versus heparin dressing in burn wound management. J Evol Med Dent Sci. 2013;2:9124-30.

12. Shettigar UR, Jagannathan R, Natarajan R. Collagen film for burn wound dressings reconstituted from animal intestines. Artif Organs. 1982;6(3):256-60.

13. Gao ZR, Hao ZQ, Li Y, Im MJ, Spence RJ. Porcine dermal collagen as a wound dressing for skin donor sites and deep partial skin thickness burns. Burns. 1992;18(6):492-6.

14. Lazovic G, Colic M, Grubor M, Jovanovic M. The Application of collagen Sheet in Open Wound Healing. Ann Burns Fire Disasters. 2005;18(3):1516.

Cite this article as: Khurram MF, Sheikh MT, Ahmad I, Maurya SK, Ambedkar V, Maurya RK. The efficacy of collagen based dressings for treatment of scald burns in under 5 years paediatric population: a noncomparative, prospective single centre experience. Int J Community Med Public Health 2018;5:4298-302. 